\title{
Overhead versus Underground: Designing Power Lines for Resilient, Cost-Effective Distribution Networks under Windstorms
}

\author{
Laiz Souto \\ Intelligent Systems \& Control Engineering group \\ Universitat de Girona \\ Girona, Spain 17002 \\ laiz.souto@gmail.com
}

\author{
Surya Santoso \\ Department of Electrical \& Computer Engineering \\ The University of Texas at Austin \\ Austin, Texas 78712-0240 \\ ssantoso@mail.utexas.edu
}

\begin{abstract}
Windstorms represent a particular class of highimpact, low-probability events that is highly likely to damage distribution poles and pull down overhead lines in vulnerable areas. As a result, when a windstorm occurs, the costs associated with damaged overhead corridors and energy not supplied may be too high. Conversely, the costs associated with the installation of underground distribution lines are expensive in comparison to overhead distribution lines and may not compensate for the penalties avoided for the loads lost. In this scenario, this article assesses the costs and risks associated with underground and overhead power lines for a resilient, cost-effective planning and operation of power distribution networks under windstorms. Thus, it calculates the accumulated costs associated with installation, operation, and repair of power distribution lines, as well as the penalties for the energy not supplied, subject to the probability of failure of individual components over time, to determine which power line setting is the most appropriate in terms of resilience and costs.
\end{abstract}

Index Terms - extreme weather, fragility curve, power distribution networks, power system resilience, uncertainty modeling

\section{INTRODUCTION}

$\mathbf{N}$ ATURAL disasters result in huge monetary losses and damages to critical infrastructures, including electric power systems. The frequency and intensity of natural disasters has increased in the last decades and is expected to rise even more in the future due to climate change, resulting in potentially catastrophic impacts to economy and society [1]. Therefore, enhancing resilience of power systems is crucial to withstand and recover from these high-impact, low-probability events, maintaining quality of supply and minimizing performance degradation.

Among all power system components, overhead power distribution lines are particularly vulnerable to outages caused

This research was supported by the European Union's Horizon 2020 research and innovation programme, call LCE-01-2016-2017, under the auspices of the project "Renewable penetration levered by Efficient Low Voltage Distribution grids", grant agreement number 773715, and University of Girona scholarship. We acknowledge that this work benefits from discussion and collaboration with the project "Defending the Electricity Infrastructure agains Extreme Weather Events, Now and in the Future", funded by The University of Texas at Austin Energy Institute. by different extreme events, for their manufacturing and assembling, insofar as they transport electricity from generation sites and distribution substations to consumers across relatively long distances. Moreover, the design principles that enable transmission lines to withstand natural disasters, such as ice and wind loads [2], do not apply to power distribution lines [3]. As a consequence, their failure rates are expected to be high in comparison with other grid components and can put the grid operation at a higher risk of collapsing under extreme weather events [4].

Among all natural disasters, strong winds represent a particular class of high-impact, low-probability events that are highly likely to damage distribution poles and pull down overhead lines in vulnerable areas. Methodologies aimed at enhancing power system resilience under strong winds have been previously presented in [5], which proposed a defensive islanding strategy, whereas [6] modeled hurricane exposure, fragility curves, and restoration costs. To this extent, [7] evaluated hardening and operational resilience strategies and [8] proposed a robust operation framework. In addition, [9]-[12] performed a quantitative assessment of critical components to prioritize resilience enhancements.

As an option, overhead lines could be replaced with underground lines to enhance the ability of the system to withstand high-impact, low-probability events and thereby reduce interruption times. Underground lines present low failure rates and cheap operational costs in comparison with overhead lines. However, this choice takes its toll with high installation costs and long restoration times in the event of an outage. Therefore, it is necessary to evaluate the pros and cons of choosing between overhead and underground power lines for a resilient, cost-effective planning and operation of power systems under extreme weather events. In this context, a methodology capable of providing support in the decisionmaking process is attractively aligned with the task. However, none of the previous efforts has compared the performance of underground versus overhead power lines in terms of resilience and costs.

Fitting into this context, this article assesses the costs 
and risks associated with installation, operation, and repair of underground and overhead power lines for a resilient, cost-effective power system planning and operation under windstorms. The penalty costs for the energy not supplied are also taken into account within the problem formulation to determine the most appropriate power line setting in terms of resilience and costs. Furthermore, the methodology is tested in different case studies based on a real power distribution network in Catalonia, Spain, modeled and simulated in MATLAB/Simulink.

The text is organized as follows. The methodology is described in Section II, the case studies are depicted in Section III, results and discussions are described in Section IV, and conclusions are finally presented in Section V.

\section{Methodology}

The choice between overhead and underground power lines is formulated as a long-term cost minimization function, considering the costs associated with the installation, operation, and repair of individual components together with their probability of failure. Thus, consider a distribution grid with $E$ lines and $N$ poles (in case of overhead lines) and let $\mathcal{X}_{i}$ be the set of overhead and underground lines available for each distribution corridor $i$, with $x_{i} \in \mathcal{X}_{i}, i=1, \ldots, E$. Let $c_{x_{i}}^{\text {inst }}$, $c_{x_{i}}^{o p}, c_{x_{i}}^{r e}$, and $c_{x_{i}}^{\text {out }}$ be the costs of installation, operation, repair, and penalty for the energy not supplied due to an outage of the $i^{\text {th }}$ corridor, $i=1, \ldots, E$, and consider a finite time horizon denoted by $\tau$.

The impact of windstorms on the system resilience is assessed through fragility modeling of individual poles and lines, whenever applicable, as in [13]. This procedure allows for a probabilistic evaluation of system outages as a function of the time-varying wind speed $w(t)$ (time dependency omitted from the equations for clarity and simplification). The fragility curves are given by (1) for a single pole and (2) for an overhead line, considering a critical speed $w_{\text {crit }}$ above which the probability of failure increases and a collapse speed $w_{\text {coll }}$ from which the failure is certain:

$$
\begin{aligned}
& P_{T}(w)=\left\{\begin{array}{l}
0, \text { if } w<w_{\text {crit }} \\
P_{T, h w}, \text { if } w_{\text {crit }} \leq w<w_{\text {coll }} \\
1, \text { if } w \geq w_{\text {coll }}
\end{array},\right. \\
& P_{L}(w)=\left\{\begin{array}{l}
P_{L, \text { std }}, \text { if } w<w_{\text {crit }} \\
P_{L, h w}, \text { if } w_{\text {crit }} \leq w<w_{\text {coll }} \\
1, \text { if } w \geq w_{\text {coll }}
\end{array}\right.
\end{aligned}
$$

The outage of each pole is supposed to be independent of the condition of adjacent poles in the $i^{t h}$ corridor (that is, the failures of different components in the same corridor are regarded as independent events and not conditioned to the status of other components). Likewise, the failure of a power line is independent from the failure of poles in the $i^{\text {th }}$ corridor. Considering that $N_{i}$ poles are connected in series along the $i^{t h}$ corridor, the failure of a single component will trip the entire corridor $i$. Let $F_{L, x_{i}}$ be the failure function of a line, equivalent to (2), and $F_{T, j}$ be the failure function of a pole, equivalent to
(1), $j=1, \ldots, N_{i}$. It holds that the joint probability of failure as a function of the wind speed, denoted by $P_{x_{i}}(w)$, is such that (3) holds as follows:

$$
P_{x_{i}}(w)=1-P\left[\left(F_{L, x_{i}}=0\right) \cap\left(F_{T, 1}=0\right) \cap \ldots \cap\left(F_{T, N_{i}}=0\right)\right],
$$

with

$$
\left\{\begin{array}{l}
P\left[F_{L, x_{i}}=0\right]=1-P_{L}(w) \\
P\left[F_{T, j}=0\right]=1-P_{T}(w), j=1, \ldots, N_{i}
\end{array}\right.
$$

For underground lines, (2) is replaced with $P_{L}(w)=P_{L, s t d}$ constant and (1) does not apply. Thereby, (3) is equivalent to $P_{x_{i}}(w)=P_{L, s t d}, \forall w$.

The wind speed profile over time is defined as a stochastic function, as in [14], following the Weibull probability distribution function $f(w)$ given by

$$
f(w)=k \frac{w^{k-1}}{c^{k}} \exp \left(-\left(\frac{w}{c}\right)^{k}\right),
$$

where $c$ is a scale parameter and $k$ is a shape parameter.

Then, considering that the cost estimates are made every hour $t$ and that $w$ is time-dependent, i.e. $w=w(t)$, the accumulated cost of a single corridor over $\tau$ is given by

$$
\begin{aligned}
c_{x_{i}}=c_{x_{i}}^{i n s t}+ & \sum_{t=1}^{\tau}\left[c_{x_{i}}^{o p}(t)+\right. \\
& \left.+P_{x_{i}}(w(t))\left(c_{x_{i}}^{r e}(w(t))+c_{x_{i}}^{\text {out }}(t)\right)\right] .
\end{aligned}
$$

In (6), $c_{x_{i}}^{\text {inst }}$ is proportional to the line length, denoted by $d_{L}$, whereas $c_{x_{i}}^{o p}$ is proportional to the contracted power of all customers and the line length divided by the total length of the network. The penalty applied for the energy not supplied is calculated with the contracted power of each affected customer (robust scenario), which is assumed to be time-independent and weather-independent. However, $c_{x_{i}}^{\text {out }}$ is time-varying due to the out-of-service loads changing over time. In turn, $c_{x_{i}}^{r e}$ is assumed to be a function of $w$ to reflect that the damage and time to repair increase with the wind speed, according to [13].

Alternatively, the value of lost load resulting from the failure of the $i^{t h}$ corridor can be denoted by $V o L L_{x_{i}}$ and calculated in separate with

$$
V o L L_{x_{i}}=\sum_{t=1}^{\tau} P_{x_{i}}(w(t)) c_{x_{i}}^{\text {out }}(t) .
$$

Considering the accumulated cost, the long-term cost minimization problem is given by (8) as follows, with $c_{i} *$ denoting the optimal solution in terms of accumulated costs for the $i^{t h}$ corridor

$$
c_{i} *=\min _{x_{i} \in \mathcal{X}_{i}} c_{x_{i}} .
$$

Alternatively, considering the value of load lost, the longterm cost minimization problem is given by (9) as follows, 
with $V o L L_{i} *$ denoting the optimal solution in terms of the value of load lost for the $i^{\text {th }}$ corridor

$$
\begin{gathered}
V o L L_{i} *=\min _{x_{i} \in \mathcal{X}_{i}} \operatorname{VoLL} L_{x_{i}} . \\
\text { III. CASE STUDY }
\end{gathered}
$$

The proposed methodology is demonstrated in a real-based rural distribution network, whose topology is illustrated in Fig. 1. In total, the network has 2 distribution substations, 24 feeders, 3 switches, and 20 different energy consumers (among them, 1 industrial, three-phase and 19 residential, single-phase). The sizing of substation transformers is 500 $\mathrm{kVA}$ at SS-1 and $250 \mathrm{kVA}$ at SS-2, whereas the contracted power is $100 \mathrm{~kW}$ for the industrial customer (power factor 0.96 ) and $10 \mathrm{~kW}$ for each single-phase residential customer (power factor 0.92).

The switches connecting the distribution substation with the external grid are normally closed, whereas the switch connecting the two parts of the distribution network is normally open. In the event of a component failure, they enable grid reconfiguration and disconnection whenever needed in order to minimize the total load shedding. The present analysis considers one component failure at a time, complying with the $N-1$ reliability criterion (in other words, it does not consider multiple component failures occurring simultaneously).

The following situations are considered in the specification of this grid: underground lines, overhead lines without hardening, and overhead lines with additional hardening. The costs and failure rates of the power lines in use are approximations from [15] and [16], whereas the cost of energy not supplied, given by the electricity price multiplied by the load lost, is an approximation from [17]. In addition, the duration of an outage (thereupon denoted by $t_{\text {out }}$ ) is assumed to be longer in underground lines than in overhead lines, in accordance to [15]. The costs are assumed to be constant over the time horizon of the analysis. For simplification, the calculations neglect capacity expansion and interest rates, as the effect on the costs associated with different line settings would be proportional to the results calculated in the reference year.

For comparison, a ten-year and twenty-year time horizon are considered to compute the costs associated with operation, repair, and out-of-service loads of the grid. In addition, penalty costs equivalent to $c_{x_{i}}^{\text {out }}=7.0 \$ / \mathrm{kWh}$ and $c_{x_{i}}^{\text {out }}=35.0 \$ / \mathrm{kWh}$ are analyzed over $\tau=10$ years. The other costs in use for individual overhead and underground lines are summarized in Table I, whereas the lengths of the lines highlighted in Fig. 1 and load shedding resulting from a failure along them are listed in Table II. The following scenarios are analyzed:

1) $\tau=10$ years and $c_{x_{i}}^{\text {out }}=7.0 \$ / \mathrm{kWh}$,

2) $\tau=20$ years and $c_{x_{i}}^{\text {out }}=7.0 \$ / \mathrm{kWh}$, and

3) $\tau=10$ years and $c_{x_{i}}^{\text {out }}=35.0 \$ / \mathrm{kWh}$

The fragility curves are given by (10) and (11) for an overhead corridor without hardening, by (10) and (12) for an overhead corridor with additional hardening along the lines, and by (13) for an underground corridor. The wind speeds are
TABLE I

COST PARAMETERS

\begin{tabular}{llll}
\hline \hline & Light Overhead & Hardened Overhead & Underground \\
\hline$c_{x_{i}}^{\text {inst }}$ & $100,000 \$ / \mathrm{mi}$ & $200,000 \$ / \mathrm{mi}$ & $400,000 \$ / \mathrm{mi}$ \\
$c_{x_{i}}^{o p}$ & $0.10 \$ / \mathrm{kWh}$ & $0.10 \$ / \mathrm{kWh}$ & $0.05 \$ / \mathrm{kWh}$ \\
$c_{x_{i}}^{r e}$ & $1,000 \$ / \mathrm{mi}$ & $2,000 \$ / \mathrm{mi}$ & $10,000 \$ / \mathrm{mi}$ \\
$t_{\text {out }}$ & $12 \mathrm{~h}$ & $12 \mathrm{~h}$ & $72 \mathrm{~h}$ \\
\hline \hline
\end{tabular}

TABLE II

LINE PARAMETERS

\begin{tabular}{llll}
\hline \hline \#Line & Length [mi] & Number of poles & Shedding [kW] \\
\hline 1 & 21 & 2 & 50 \\
2 & 28 & 3 & 10 \\
3 & 36 & 4 & 0 \\
4 & 42 & 4 & 0 \\
5 & 25 & 3 & 0 \\
6 & 36 & 4 & 0 \\
7 & 15 & 2 & 0 \\
8 & 42 & 4 & 30 \\
\hline \hline
\end{tabular}

measured in miles per hour, as the line lengths are given in miles.

$$
\begin{gathered}
P_{T}(w)=\left\{\begin{array}{l}
0, \text { if } w<40 \\
\frac{w-40}{60-40}, \text { if } 40 \leq w<60 \\
1, \text { if } w \geq 60
\end{array}\right. \\
P_{L 1}(w)=\left\{\begin{array}{l}
\frac{1.25 \times d_{L}}{8760} h^{-1}, \text { if } w<25 \\
\frac{w-25}{40-25}\left(1-\frac{1.25 \times d_{L}}{8760}\right)+\frac{1.25 \times d_{L}}{8760}, \text { if } 25 \leq w<40 \\
1, \text { if } w \geq 40
\end{array}\right. \\
P_{L 2}(w)=\left\{\begin{array}{l}
\frac{0.75 \times d_{L}}{8760} h^{-1}, \text { if } w<40 \\
\frac{w-40}{60-40}\left(1-\frac{0.75 \times d_{L}}{8760}\right)+\frac{0.75 \times d_{L}}{8760}, \text { if } 40 \leq w<60 \\
1, \text { if } w \geq 60
\end{array}\right. \\
P_{L 3}(w)=\frac{0.25 \times d_{L}}{8760} h^{-1}, \forall w
\end{gathered}
$$

The probability distribution function of the wind speed is calculated with (5) using $c=10$ and $k=1.2$. The wind speed over time is drawn randomly from (5) using MATLAB. A one-week interval is considered to calculate the costs over the outage times defined in Table I.

\section{RESUlTS AND DISCUSSION}

This section presents the results calculated for the scenarios described in Section III. The value of load lost and the accumulated costs calculated for overhead lines without hardening, with hardening, and underground lines are shown in Tables III and IV for $\tau=10$ years and Tables V and VI for $\tau=20$ years with $c_{x_{i}}^{\text {out }}=7.0 \mathrm{US} \$ / \mathrm{kWh}$ and in Tables VII and VIII for $\tau=10$ years with $c_{x_{i}}^{\text {out }}=35.0 \mathrm{US} \$ / \mathrm{kWh}$. Additionally, Fig. 2 illustrates the chosen lines in terms of accumulated costs according to the scenarios enumerated in Section III. 


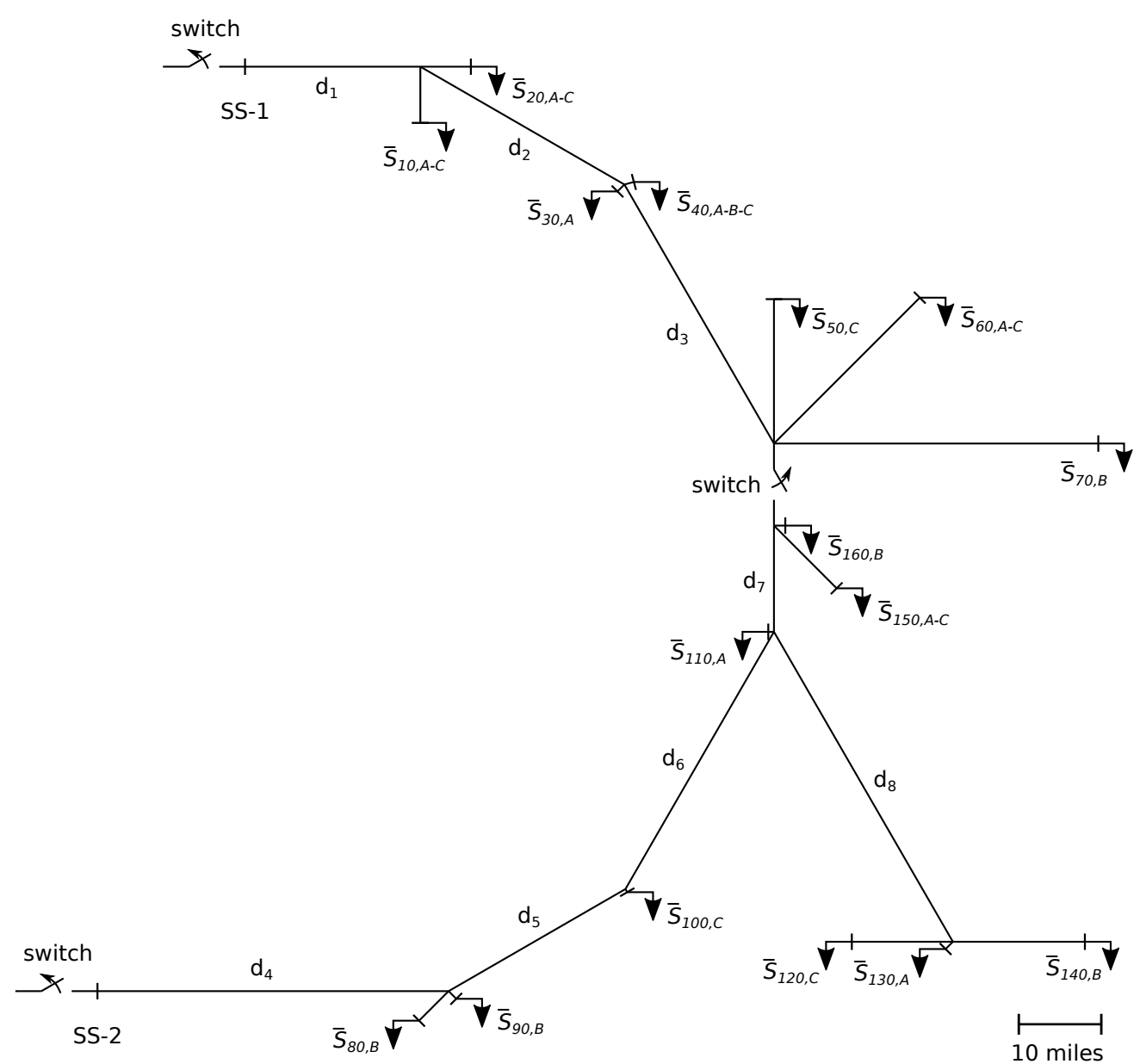

Fig. 1. Single-line diagram of the radial distribution network, with 2 distribution substations (SS-1 and SS-2), 24 feeders drawn to scale, 3 switches, 1 industrial three-phase customer $\left(\bar{S}_{40, A-B-C}\right)$, and 19 residential single-phase customers $\left(\bar{S}_{10, A}, \bar{S}_{10, C}, \bar{S}_{20, A}, \bar{S}_{20, C}, \bar{S}_{30, A}, \bar{S}_{50, C}, \bar{S}_{60, A}, \bar{S}_{60, C}\right.$, $\left.\bar{S}_{70, B}, \bar{S}_{80, B}, \bar{S}_{90, B}, \bar{S}_{100, C}, \bar{S}_{110, A}, \bar{S}_{120, C}, \bar{S}_{130, A}, \bar{S}_{140, B}, \bar{S}_{150, A}, \bar{S}_{150, C}, \bar{S}_{160, B}\right)$.

TABLE III

RESULTS: VALUE OF LOAD LOST WITH $\tau=10$ YEARS AND $c_{x_{i}}^{\text {out }}=7.0 \$ / \mathrm{KWH}(\mathrm{IN} \$)$

\begin{tabular}{llll}
\hline \hline$i$ & Light Overhead & Hardened Overhead & Underground \\
\hline 1 & 29,343 & 4,142 & 7 \\
2 & 5,869 & 895 & 2 \\
3 & 0 & 0 & 0 \\
4 & 0 & 0 & 0 \\
5 & 0 & 0 & 0 \\
6 & 0 & 0 & 0 \\
7 & 0 & 0 & 0 \\
8 & 17,610 & 2,825 & 9 \\
\hline \hline
\end{tabular}

It can be noticed that the overhead corridors without hardening present the highest values of load lost when a failure causes load shedding, whereas underground lines present the lowest values of load lost when a failure causes load shedding in all simulations. This evinces the high impact of windstorms on overhead corridors in terms of damage and load lost.

On the other hand, the accumulated costs calculated with (6) are the lowest for overhead lines without hardening and the highest for underground cables for all corridors when when
TABLE IV

RESULTS: ACCUMULATED COSTS WITH $\tau=10$ YEARS AND $c_{x_{i}}^{\text {out }}=7.0 \$ / \mathrm{KWH}(\mathrm{IN} \$)$

\begin{tabular}{llll}
\hline \hline Number & Light Overhead & Hardened Overhead & Underground \\
\hline 1 & 31,807 & 8,601 & 8,517 \\
2 & 9,155 & 6,845 & 11,347 \\
3 & 4,224 & 7,653 & 14,472 \\
4 & 4,928 & 8,929 & 17,018 \\
5 & 2,933 & 5,312 & 10,129 \\
6 & 4,224 & 7,653 & 14,472 \\
7 & 1,760 & 3,185 & 6,078 \\
8 & 22,539 & 11,754 & 17,027 \\
\hline \hline
\end{tabular}

a component failure produces no load shedding (that is, all lines except 1,2 , and 8 in all scenarios). Conversely, for line number $1,(6)$ is the lowest with use of underground cables for all scenarios due to the relatively high load shedding; for line number $2,(6)$ is the lowest with use of overhead lines with hardening for all scenarios due to the relatively low load shedding; and for line number $8,(6)$ is the lowest with use of overhead lines with hardening when $c_{x_{i}}^{\text {out }}=7.0 \mathrm{US} \$ / \mathrm{kWh}$ and the lowest with use of underground cables when the penalty is 


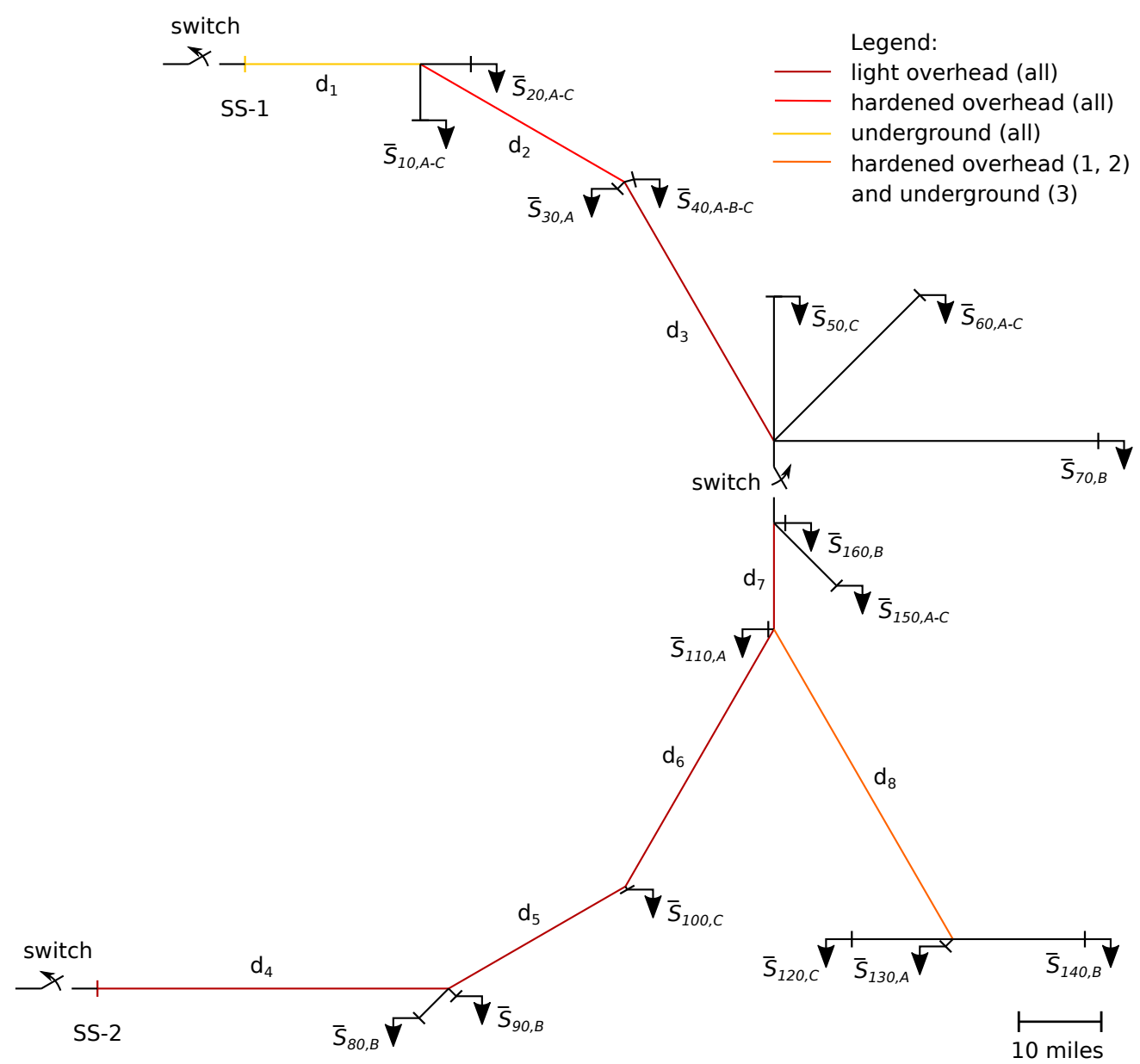

Fig. 2. Single-line diagram of the radial distribution network showing the lines chosen in terms of accumulated costs.

TABLE V

RESULTS: VALUE OF LOAD LOST WITH $\tau=20$ YEARS AND $c_{x_{i}}^{\text {out }}=7.0 \$ / \mathrm{KWH}(\mathrm{IN} \$)$

\begin{tabular}{llll}
\hline \hline Number & Light Overhead & Hardened Overhead & Underground \\
\hline 1 & 57,181 & 8,319 & 15 \\
2 & 11,437 & 1,735 & 4 \\
3 & 0 & 0 & 0 \\
4 & 0 & 0 & 0 \\
5 & 0 & 0 & 0 \\
6 & 0 & 0 & 0 \\
7 & 0 & 0 & 0 \\
8 & 34,316 & 5,348 & 18 \\
\hline \hline
\end{tabular}

TABLE VI

RESULTS: ACCUMULATED COSTS WITH $\tau=20$ YEARS AND $c_{x_{i}}^{\text {out }}=7.0 \$ / \mathrm{KWH}(\mathrm{IN} \$)$

\begin{tabular}{llll}
\hline \hline Number & Light Overhead & Hardened Overhead & Underground \\
\hline 1 & 60,002 & 13,037 & 8,634 \\
2 & 15,199 & 8,031 & 11,494 \\
3 & 4,837 & 8,099 & 14,774 \\
4 & 5,643 & 9,449 & 17,236 \\
5 & 3,359 & 5,622 & 10,260 \\
6 & 4,837 & 8,099 & 14,774 \\
7 & 2,015 & 3,373 & 6,156 \\
8 & 39,959 & 14,797 & 17,255 \\
\hline \hline
\end{tabular}

TABLE VII

RESULTS: VALUE OF LOAD LOST WITH $\tau=10$ YEARS AND $c_{x_{i}}^{\text {out }}=35.0 \$ / \mathrm{KWH}(\mathrm{IN} \$)$

\begin{tabular}{llll}
\hline \hline$i$ & Light Overhead & Hardened Overhead & Underground \\
\hline 1 & 146,720 & 20,710 & 39 \\
2 & 29,345 & 4,476 & 10 \\
3 & 0 & 0 & 0 \\
4 & 0 & 0 & 0 \\
5 & 0 & 0 & 0 \\
6 & 0 & 0 & 0 \\
7 & 0 & 0 & 0 \\
8 & 88,049 & 14,122 & 47 \\
\hline \hline
\end{tabular}

TABLE VIII

RESULTS: ACCUMULATED COSTS WITH $\tau=10$ YEARS AND $c_{x_{i}}^{\text {out }}=35.0 \$ / \mathrm{KWH}(\mathrm{IN} \$)$

\begin{tabular}{llll}
\hline \hline Number & Light Overhead & Hardened Overhead & Underground \\
\hline 1 & 149,184 & 25,168 & 8,548 \\
2 & 32,631 & 10,425 & 11,356 \\
3 & 4,224 & 7,653 & 14,587 \\
4 & 4,929 & 8,930 & 17,018 \\
5 & 2,933 & 5,312 & 10,129 \\
6 & 4,224 & 7,653 & 14,587 \\
7 & 1,760 & 3,185 & 6,078 \\
8 & 92,979 & 23,052 & 17,065 \\
\hline \hline
\end{tabular}


increased to $c_{x_{i}}^{\text {out }}=35.0 \mathrm{US} \$ / \mathrm{kWh}$ due to the costs associated with load shedding in different scenarios.

It is noteworthy that the small values of load lost calculated for the underground lines reflect the small failure rate of these components and their reduced length, as well as the load demand and the time horizon of the analysis. Unlike overhead lines, underground lines do not face outages caused by strong winds, which result in with longer interruption times and higher costs associated with the out-of-service loads. Were higher failure rates to be used, the value of loads lost would be more expensive for underground lines.

To conclude, the results indicate that the accumulated costs associated with operation, repair, and penalties for the energy not supplied may compensate the installation costs in a long time horizon. In some cases, depending on the length of the line and load shedding caused by a failure, the installation of more reliable, but expensive power lines may be the best option when it comes to both resilience increase and cost reduction in the long run. Furthermore, the results suggest that a high penalty for the energy not supplied may be an effective policy mechanism to incentive resilience-, cost-effective power system planning.

\section{Conclusion}

This article has assessed the costs and risks associated with underground and overhead power lines for a resilient, cost-effective power system planning and operation under windstorms. The method evaluated the cost associated with installation, operation, and repair of power lines, as well as the penalties for the energy not supplied (value of lost load) over the time horizon of the analysis, to determine which power line setting is the most appropriate in terms of resilience and costs for different case studies. It also considers the failure probability as a function of the wind speed and fragility modeling of individual components for a probabilistic impact assessment. Then, for each distribution corridor, the algorithm searches for the alternative which presents the minimum accumulated costs and/or value of load lost. The results show that there is a trade-off between resilience and costs, as the installation costs are the highest for underground lines, whereas the value of lost load is the highest for overhead lines without hardening in most cases.

\section{REFERENCES}

[1] C. Field, V. Barros, T. Stocker, D. Qin, D. Dokken, K. Ebi, M. Mastrandrea, K. Mach, G.-K. Plattner, S. Allen, M. Tignor, and P. M. (eds), Managing the Risks of Extreme Events and Disasters to Advance Climate Change Adaptation. A Special Report of Working Groups I and II of the Intergovernmental Panel on Climate Change. Cambridge University Press, 2012.

[2] A. Goel, Design of Transmission Lines for Atmospheric Icing. Dordrecht: Springer Netherlands, 2008, pp. 327-371.

[3] M. F. Jurgemeyer and B. M. Miller, "Nesc wind and ice load effects on wood distribution pole design," IEEE Transactions on Industry Applications, vol. 50, no. 5, pp. 3004-3010, 2014.

[4] B. Vaagensmith, T. McJunkin, K. Vedros, J. Reeves, J. Wayment, L. Boire, C. Rieger, and J. Case, "An integrated approach to improving power grid reliability: Merging of probabilistic risk assessment with resilience metrics," in 2018 Resilience Week (RWS), Aug 2018, pp. 139146.
[5] M. Panteli, D. N. Trakas, P. Mancarella, and N. D. Hatziargyriou, "Boosting the power grid resilience to extreme weather events using defensive islanding," IEEE Transactions on Smart Grid, vol. 7, no. 6, pp. 2913-2922, Nov 2016.

[6] E. B. Watson and A. H. Etemadi, "Modeling electrical grid resilience under hurricane wind conditions with increased solar and wind power generation," IEEE Transactions on Power Systems, vol. 35, no. 2, pp. 929-937, March 2020.

[7] M. Panteli, D. N. Trakas, P. Mancarella, and N. D. Hatziargyriou, "Power systems resilience assessment: Hardening and smart operational enhancement strategies," Proceedings of the IEEE, vol. 105, no. 7, pp. 1202-1213, July 2017.

[8] M. Esfahani, N. Amjady, B. Bagheri, and N. D. Hatziargyriou, "Robust resiliency-oriented operation of active distribution networks considering windstorms," IEEE Transactions on Power Systems, pp. 1-1, 2020.

[9] D. N. Trakas, N. D. Hatziargyriou, M. Panteli, and P. Mancarella, "A severity risk index for high impact low probability events in transmission systems due to extreme weather," in 2016 IEEE PES Innovative Smart Grid Technologies Conference Europe (ISGT-Europe), Oct 2016, pp. 16.

[10] J. W. Muhs and M. Parvania, "Stochastic spatio-temporal hurricane impact analysis for power grid resilience studies," in 2019 IEEE Power Energy Society Innovative Smart Grid Technologies Conference (ISGT), Feb 2019, pp. 1-5.

[11] Y. Yang, W. Tang, Y. Liu, Y. Xin, and Q. Wu, "Quantitative resilience assessment for power transmission systems under typhoon weather," IEEE Access, vol. 6, pp. 40747-40756, 2018.

[12] G. Li, G. Huang, Z. Bie, Y. Lin, and Y. Huang, "Component importance assessment of power systems for improving resilience under wind storms," Journal of Modern Power Systems and Clean Energy, vol. 7, no. 4, pp. 676-687, July 2019.

[13] M. Panteli, C. Pickering, S. Wilkinson, R. Dawson, and P. Mancarella, "Power system resilience to extreme weather: Fragility modeling, probabilistic impact assessment, and adaptation measures," IEEE Transactions on Power Systems, vol. 32, no. 5, pp. 3747-3757, Sep. 2017.

[14] T. Yeh and L. Wang, "A study on generator capacity for wind turbines under various tower heights and rated wind speeds using weibull distribution," IEEE Transactions on Energy Conversion, vol. 23, no. 2, pp. 592-602, June 2008 .

[15] W. Warwick, T. Hardy, M. Hoffman, and J. Homer, "Electricity distribution system baseline report," July 2016.

[16] F. Roos and S. Lindahl, "Distribution system component failure rates and repair times - an overview," 2004. [Online]. Available: http://lup.lub.lu.se/record/694718

[17] London Economics International LLC, "Estimating the value of lost load," July 2013. 